\title{
Potent anti-tumor killing activity of the multifunctional Treg cell line HOZOT against human tumors with diverse origins
}

\author{
TOSHIYA INOUE $^{1 *}$, YUICHI TASHIRO ${ }^{2 *}$, MAKOTO TAKEUCHI $^{1}$, TAKESHI OTANI ${ }^{1}$, KAZUE TSUJI-TAKAYAMA $^{1}$, \\ AYUMI OKOCHI ${ }^{1}$, YURIKO MUKAE ${ }^{2}$, MAYUKO KOREISHI ${ }^{2}$, FUMIYUKI YAMASAKI $^{4}$, HIROMI KUMON ${ }^{3}$, \\ SHUJI NAKAMURA ${ }^{1}$, MASAYOSHI KIBATA ${ }^{1}$ and EISAKU KONDO ${ }^{2,5}$ \\ ${ }^{1}$ Cell Biology Institute, Research Center, Hayashibara Biochemical Laboratories Inc., 675-1, Fujisaki, Naka-ku, \\ Okayama 702-8006; Departments of ${ }^{2}$ Pathology and ${ }^{3}$ Urology, Okayama University Graduate School of \\ Medicine, Dentistry and Pharmaceutical Sciences, 2-5-1, Shikata-cho, Kita-ku, Okayama 700-8558; ${ }^{4}$ Kurashiki \\ Medical Center, 250, Bakuro-cho, Kurashiki, Okayama 710-8522; 5 Division of Oncological Pathology, \\ Aichi Cancer Center Research Institute, 1-1 Kanokoden, Chikusa-ku, Nagoya 464-8681, Japan
}

Received November 17, 2010; Accepted December 29, 2010

DOI: 10.3892/ijo.2011.962

\begin{abstract}
The T cell line HOZOT has a unique FOXP $3+{ }^{+} \mathrm{CD} 4^{+}$ $\mathrm{CD} 8{ }^{+} \mathrm{CD} 25^{+}$phenotype, exhibits suppressive activity in allogeneic mixed lymphocyte reactions (MLR), and produces IL-10, defining HOZOT as regulatory T cells (Tregs). Interestingly, in addition to possessing a suppressive Treg ability, HOZOT was also found to show cytotoxicity against certain representative human cancer cell types. In order to disclose the range of anti-tumor activity by HOZOT, we screened it by using a panel of twenty human tumor cell lines with different origins. Consequently, HOZOT showed potent cytocidal effects against a wide spectrum of neoplastic cells including carcinomas, sarcomas, mesotheliomas and glioblastomas except for hematopoietic malignancies. Its anti-tumor activity was strong enough with an E:T ratio of 4:1, which is considered to be more effective than that by conventional CTLs. Furthermore,
\end{abstract}

Correspondence to: Dr Toshiya Inoue, Cell Biology Institute, Research Center, Hayashibara Biochemical Laboratories Inc., 675-1, Fujisaki, Naka-ku, Okayama 702-8006, Japan

E-mail: t_inoue@hayashibara.co.jp

Dr Eisaku Kondo, Division of Oncological Pathology, Aichi Cancer Center Research Institute, 1-1 Kanokoden, Chikusa-ku, Nagoya 464-8681, Japan

E-mail: ekondo@aichi-cc.jp

*Contributed equally

Abbreviations: MLR, mixed lymphocyte reaction; Treg, regulatory T cell; UCB, umbilical cord blood; MNC, mononuclear cell; NHDF, normal human dermal fibroblast; GFP, green fluorescent protein; PBMC, peripheral blood mononuclear cell; CMA, concanamycin A; FITC, fluorescein isothiocyanate

Key words: cytotoxic treg, immunotherapy, in vivo, NOD/SCID mouse an in vivo representative mouse tumor model by implanting human colon adenocarcinoma cells revealed that adoptive transfer of HOZOT almost completely eradicated disseminated lesions on peritoneum, markedly reduced metastases in lung and liver, and dramatically decreased bloody ascites caused by peritoneal carcinomatosis. Treatment of the tumor model mice by HOZOT with an E:T ratio of 2:1 even indicated the prolongation of their survival, although not reaching obvious statistical significance. In vitro blocking experiments using antibodies and inhibitors suggested that the cytotoxic mechanism of HOZOT against tumors is different from conventional cytotoxic cells such as CTL, NK or NKT cells. Altogether, our studies demonstrated the potent killing activity of HOZOT against a broad range of human malignancies, which indicates that HOZOT is a powerful tool in immunotherapy for advanced stage tumors.

\section{Introduction}

Our previous report characterized the novel Treg cell line HOZOT (1), which was established from umbilical cord blood (UCB) mononuclear cells (MNC) by cocultivation with mouse stromal cells. HOZOT cells were uniquely multifunctional as they exhibited both suppressor and cytotoxic activities. Interestingly, HOZOT was cytotoxic not only for the xenogeneic mouse cells used for initiating the cell line but also for a number of unrelated human cancer cells. This unexpected anti-tumor activity prompted us to investigate the possible application of HOZOT as a cellular therapy for tumors.

A number of different immune cells have been characterized as anti-tumor effector cells, such as $\mathrm{CD} 8^{+}$cytotoxic $\mathrm{T}$ cells (2), $\gamma / \delta$ T cells (3), NK cells (4), and NKT (5) cells. Conversely, Tregs play an opposing role in tumor immunology, often encouraging the growth of tumors in the microenvironment because of their immunosuppressive effects on anti-tumor effector cells (6). A great deal of effort has been focused on eliminating or reducing the immunosuppressive ability of Tregs in order to promote tumor regression (2). 
However, we reasoned that because of its unique $\mathrm{CD} 4^{+} \mathrm{CD} 8^{+}$ phenotype and cytokine signature (high production of IFN- $\gamma$ and TNF- $\alpha$ ), HOZOT is a novel $\mathrm{T}$ cell subset distinct from conventional Tregs such as FOXP3 ${ }^{+}$natural Tregs or induced Tregs. Moreover, even well-characterized anti-tumor effectors, such as NK and NKT cells, also exhibit regulatory activities under certain circumstances, suggesting that regulatory and anti-tumor activities might not always be mutually exclusive $(7,8)$.

There are several examples of Tregs with cytotoxic activity, including $\mathrm{CD}^{+}, \mathrm{CD}^{+}$, and double negative types. For example, stimulation with anti-CD3/CD46 antibodies rendered natural Tregs cytotoxic for autologous $\mathrm{CD}^{+}, \mathrm{CD}^{+}$, and $\mathrm{CD} 14^{+}$target cells, and the cytotoxicity observed was associated with the induction of granzyme A (9). Human CD8 ${ }^{+}$Tregs induced as novel Treg subsets with CD40-activated B cells were shown to have cytotoxicity against allogenic and autologous lymphocytes through mechanisms mediated by soluble molecules and the Fas/FasL pathway (10). CD94/NKG2A-expressing CD8 ${ }^{+}$ $\mathrm{T}$ cells expanded from G-CSF-mobilized peripheral blood mononuclear cells were shown to exhibit both cytolytic and regulatory activities (11), indicating the involvement of these cells in promoting graft-versus-leukemia/tumor effects and suppressing graft-versus-host disease in stem cell transplantation. Double negative Tregs, which represent $1-5 \%$ of peripheral $\mathrm{T}$ lymphocytes, can inhibit immune response through directly killing effector T cells in an antigen-specific manner (12). The target cells of these cytotoxic Tregs are both normal immune cells and malignant cells. Although these studies substantiate the concept of cytotoxic Tregs as potent anti-tumor effectors, the significance of their cytotoxic activity has been focused mainly on the suppressor mechanism but not on the potential for practical application in tumor immunotherapy.

In this report, we first screened a variety of human tumor cell lines with diverse origins for their susceptibility to HOZOT's cytotoxic activity, and then evaluated the anti-tumor effect of HOZOT in vivo using a tumor-bearing NOD/SCID mouse xenograft model, which indicates the potential utility and preclinical value of HOZOT in the future cellular therapy. We also investigated the cytotoxic characteristics of HOZOT in in vitro blocking experiments and compared the killing mechanism with that of other conventional anti-tumor effectors, including both the target recognition and effector phases.

\section{Materials and methods}

Maintenance and establishment of cell lines. Normal mouse stromal cell line (ST2), and human cancer cell lines were cultured in RPMI-1640 medium (Sigma, St. Louis, MO) supplemented with 10\% FBS (Hyclone, South Logan, UT), $100 \mathrm{U} / \mathrm{ml}$ penicillin and $50 \mu \mathrm{g} / \mathrm{ml}$ streptomycin. This medium was used as standard culture medium. A stably green fluorescent protein (GFP)-expressing WiDr clone, WiDr-EGFP-9, was established as follows. WiDr was transfected with a pEF-EGFP plasmid and was selected with $100 \mu \mathrm{g} / \mathrm{ml}$ of G418 (Invitrogen, Carlsbad, CA). The pEF-EGFP plasmid was constructed by inserting enhanced GFP (EGFP) cDNA from pEGFP-N1 (Clontech, Mountain View, CA) into the expression vector pRER with expression driven by the EF-1 $\alpha$ promoter (13). The WiDr-EGFP-9 was maintained in standard culture medium.
HOZOT cell lines were established in our laboratory as previously described (1). HOZOT cells were maintained by coculturing with mouse stromal cells that were used for the initial establishment in standard culture medium containing $10 \mathrm{ng} / \mathrm{ml}$ recombinant human IL-2 (Peprotec). We have established over 20 different HOZOT cell lines and HOZOT-4 was used as a representative cell line in this study. All HOZOT cell lines share the basic phenotypic and functional properties as previously described (1). CD4 ${ }^{+} \mathrm{T}$ cells used for control $\mathrm{T}$ cells were prepared from $\mathrm{UCB}$-derived $\mathrm{CD} 4^{+}$cells as described previously (14). Human peripheral blood was obtained from healthy volunteers in compliance with Institutional Review Board approval. Informed consent of all donors was obtained according to the Declaration of Helsinki.

Antibodies and inhibitors. Antibodies were purchased from the following companies: NKG2D (clone 149810), TRAIL (clone 124723), TWEAK (Goat polyclonal antibody) and Fas ligand (clone 100419) from R\&D Systems, Inc.; NKp30 (clone P30-15) from Biolegend (San Diego, CA); CD8 (clone RPA-T8) from eBioscience (San Diego, CA); CD1d (clone 3C11) from BD Biosciences (San Diego, CA). Anti-TNF- $\alpha$ antibody was prepared in our laboratory. Inhibitors of kinases such as Src family kinase (PP2), p38 MAP kinase (SB202190), JNK kinase (SP600125), ERK1/2 kinases (PD98059), PI3 kinase (Wortmannin), as well as inhibitors of Caspase-3 (Z-DEVDFMK) and lytic granule exocytosis (Concanamycin A; CMA) were purchased from Calbiochem (La Jolla, CA). Antibodies or inhibitors were added to cocultivated target and effector cells.

Time-lapse live cell imaging of HOZOT cocultivated with target WiDr cells. Time-dependent movement of HOZOT cells in coculture with WiDr was monitored by a time-lapse live cell imaging system using a fluorescence microscope with a $\mathrm{CO}_{2}$ chamber and a CCD camera (IX81-ZDC system, Olympus, Tokyo, Japan). HOZOT cells were labeled with PKH-26 (red color) according to the manufacturer's instructions (Sigma Aldrich Japan, Tokyo, Japan), and WiDr-EGFP-9 was employed as a target. Twelve hours after seeding WiDr-EGFP-9 $\left(1 \times 10^{6}\right)$ in a $3.5 \mathrm{~cm}$-diameter Petri dish, PKH-26-labeled HOZOT cells $\left(2 \times 10^{6}\right)$ were added at an effector: target (E:T) ratio of 2:1. The movement of living HOZOT cells was tracked for $30 \mathrm{~h}$ and recorded. Fluorescence images at the time points shown in Fig. 1 were obtained from the video data.

Cytotoxicity assay. Human cancer cell lines were used as the target cells for cytotoxicity assays. The cytotoxic activity of HOZOT was estimated by counting living target cells after cocultivation with HOZOT by methylene blue uptake as previously described (1). Cytotoxicity was calculated as follows: Cytotoxicity $(\%)=100 \times$ (absorbance without effector-absorbance with effector) / (absorbance without effector - absorbance of blank).

In vivo anti-tumor activity of HOZOT. All animal procedures were in accordance with the Declaration of Helsinki and were approved by the Committee of Animal Rights in our institute. Female 5-week-old NOD/SCID mice (NOD.CB17-Prkdc ${ }^{\text {scid } / J}$ ) were purchased from Charles River Japan (Yokohama, Japan) 
A

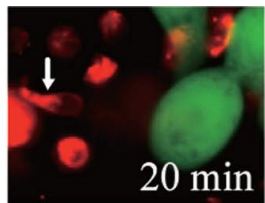

B
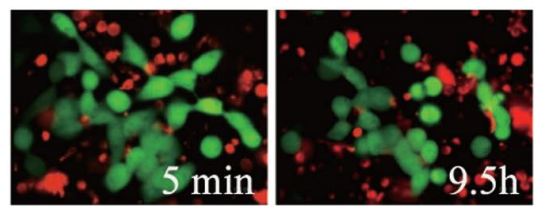

$\mathrm{C}$

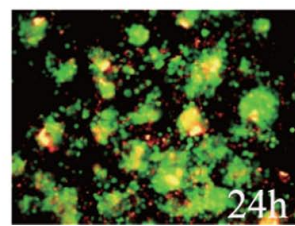

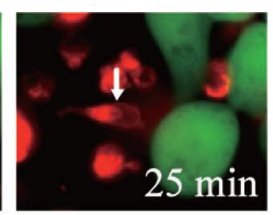
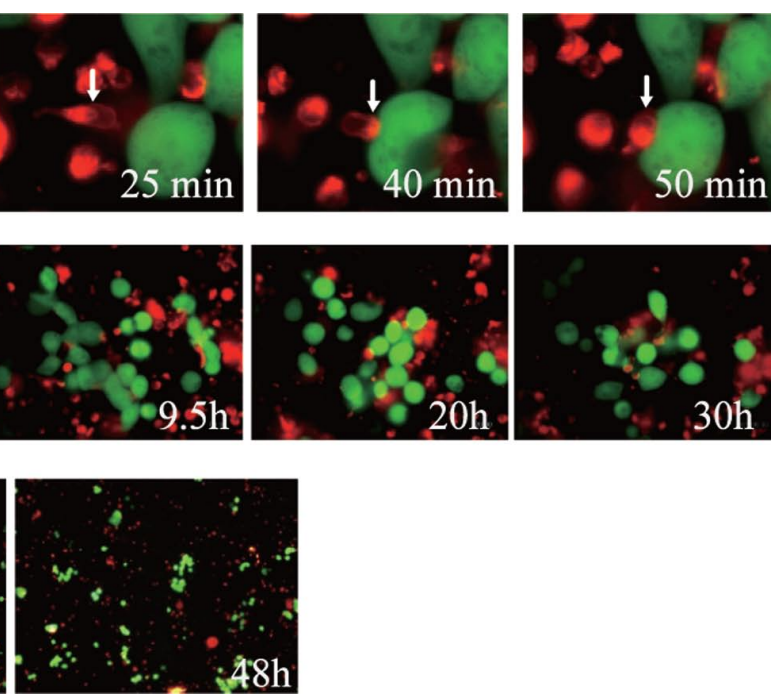

Figure 1. Cellular dynamics of HOZOT toward target cancer cells. (A) Time-lapse living cell images depicting cellular movements of PHK-26-labeled HOZOT (red) toward WiDr-EGFP-9 targets (green). Arrow depicts one of the HOZOT cells approaching and attaching to a WiDr cell. Each time indicates the addition of HOZOT to cultured WiDr cells at an E:T ratio of 2:1. (B) Dissociation of WiDr-EGFP-9 by interaction of HOZOT. Time-lapse images showing that WiDr cells become dissociated following attack by HOZOT in a time-dependent manner. Each time indicates the period after HOZOT was added. (C) Decrease of GFPexpressing WiDr cells targeted by HOZOT. Living cell images of WiDr-EGFP-9 mixed with HOZOT at 24 and 48 h. Note a remarkable decrease of WiDr cells at $48 \mathrm{~h}$.

and maintained in our animal facility under SPF conditions. A WiDr-bearing NOD/SCID mouse model was used for in vivo anti-tumor experiments. WiDr-EGFP-9 cells $\left(1 \times 10^{7}\right.$ cells/ mouse) were inoculated in the peritoneal cavity of NOD/SCID mice. After 14 days, HOZOT cells $\left(2 \times 10^{7}\right.$ cells/mouse) were inoculated in the peritoneal cavity three times every seven days. For the estimation of cytotoxic activity of HOZOT against WiDr, seven days after the final HOZOT treatment, mice were sacrificed and the remaining WiDr cells in the peritoneal or thoracic cavity were identified by fluorescence stereomicroscopy. In addition, various organs were harvested at that time and embedded in paraffin, sectioned, and stained with hematoxylin and eosin (Experiment 1). For the estimation of survival rate, WiDr-bearing NOD/SCID mice were treated with HOZOT or $\mathrm{CD}^{+}{ }^{+} \mathrm{T}$ cells for four times every seven days, and maintained until mice died due to tumor growth. For the estimation of the ascites-reduction effect, mice were monitored until they died from tumor growth, then tumoral ascites were collected and the volume was measured (Experiment 2).

Statistical analysis. Statistical analysis of in vitro cytotoxicity assays was performed using the Turkey-Kramer method. Statistical analysis of the survival rate in in vivo experiments was performed using Kaplan-Meier tests. The results were considered significantly different at $\mathrm{P}<0.05$.

\section{Results}

In vitro cellular dynamics of HOZOT and WiDr. Since we previously found that HOZOT exhibits strong cytotoxic activity against the human colorectal adenocarcinoma cell line, WiDr (1), we monitored the HOZOT movement toward the target WiDr cell line by time-lapse fluorescence microscopy. HOZOT started migrating towards the target cells and finished attaching to them within 50 min of cocultivation (depicted by arrow;
Fig. 1A), followed by marked dissociation and a decrease in the number of WiDr cells approximately $30 \mathrm{~h}$ after mixing (Fig. 1B). Forty-eight hours later, a more prominent decrease in the number of WiDr cells was observed, whereas most of the HOZOT cells were still alive (Fig. 1C).

HOZOT exhibits anti-tumor activity against a variety of cancer cell lines but not non-neoplastic human cells. Based on the preceding results using WiDr cells, we next explored if HOZOT's cytotoxic effects are a universal property of HOZOT against human malignant cells using a panel of various tumor cell lines with different origins and in vitro coculture assays. The tumor cell line panel included the following cell lines: four colorectal adenocarcinomas (Colo320DM, LoVo, WiDr and SW620), one gastric adenocarcinoma (MKN-45), one malignant melanoma (G-361), three mammary invasive ductal carcinoma cell lines (MCF-7, T47D and ZR-75-1), two ovary cystadenocarcinoma (OMC-3 and JHOS-2), four malignant mesothelioma cell lines (15) (H28, MSTO-211H, H2052 and H2452), one osteosarcoma (U2OS), one glioblastoma (U87) and one uterine cervix squamous cell carcinoma (HeLa). An embryonic kidney cell line, 293T, which is immortalized by expression of the ST40 large T antigen, was also used as a transformed target cell. We used NHDF cells as a normal human cell control. Remarkably, HOZOT exhibited considerable levels of cytotoxicity against all tested malignant tumors and transformed cell lines at E:T ratios of 4:1, with the singular exception of JHOS-2 (Fig. 2A). Although cytotoxicity levels varied from 35 to $98 \%$, the majority were $>70 \%$. HOZOT was effective in particular against carcinomas originating from the digestive tract and breast. Three mesothelioma and one hepatocellular carcinoma exhibited decreased (35-50\%) cytotoxicity in comparison with the cancers mentioned above. Noticeably, HOZOT was not cytotoxic against NHDF cells at an E:T ratio of $4: 1$, in contrast to the cases observed in tumor cell lines. To 

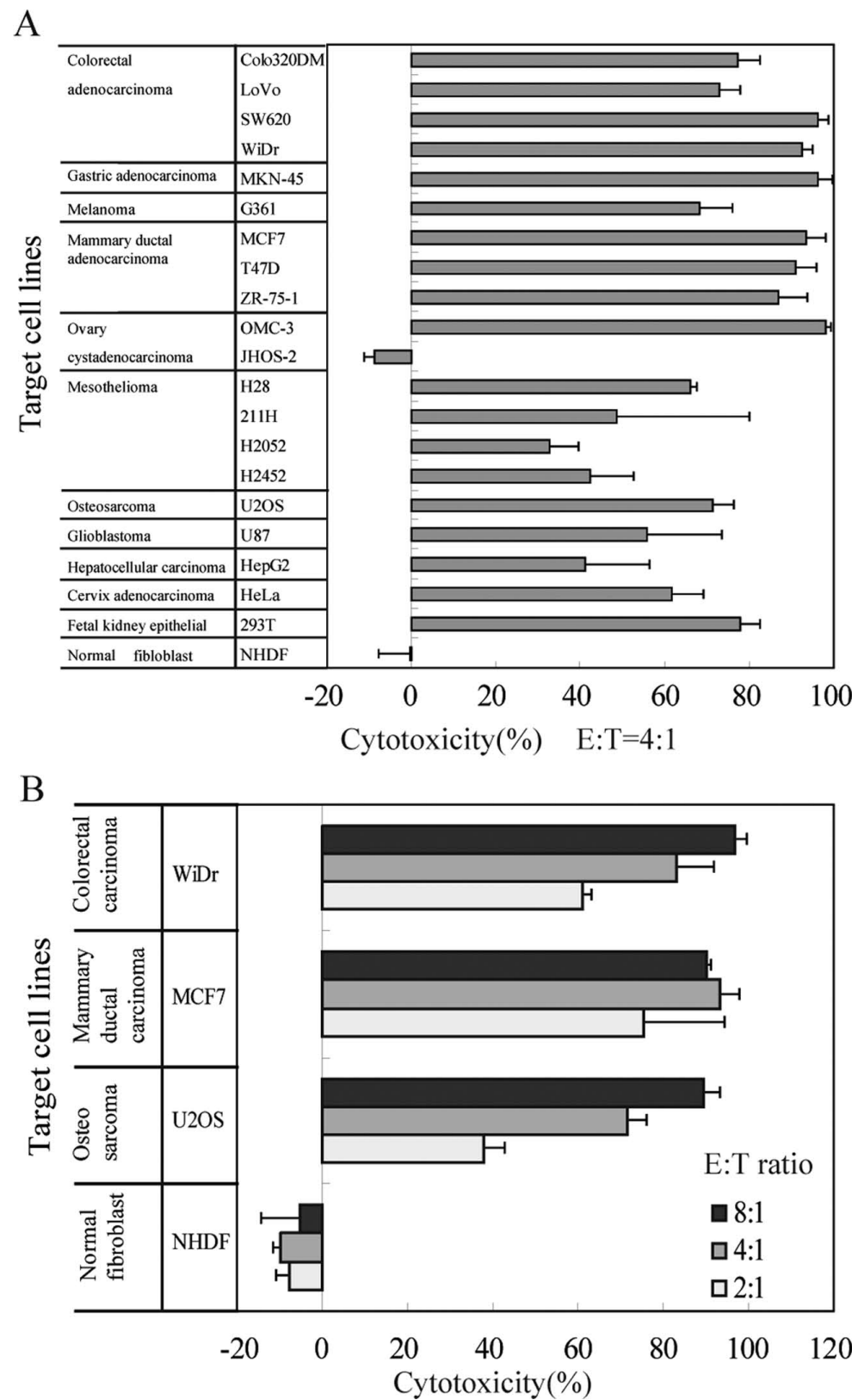

Figure 2. Cytotoxic activity of HOZOT against a broad spectrum of tumor cell lines. (A) HOZOT cytotoxicity against a variety of human normal and cancer cells. HOZOT cells were incubated with target cells at a ratio of $4: 1$ for $20 \mathrm{~h}$ and cytotoxic activity was determined by methylene blue uptake. NHDF were used as a normal cell control. HOZOT exhibited significant cytotoxicity against all examined cancer cell lines except JHOS-2, and no cytotoxicity against NHDF. (B) Dose-dependency of HOZOT cytotoxicity against normal or cancer cells. HOZOT cells were incubated with target cells at the indicated E:T ratios for $20 \mathrm{~h}$ and cytotoxicity was determined as described above. HOZOT exhibited more than $90 \%$ cytotoxicity against cancer cell lines, but had no cytotoxicity against NHDF at an E:T ratio of 8:1.

confirm HOZOT's tumor-specific cytotoxicity, we next tested its cytotoxicity at different E:T ratios. As shown in Fig. 2B, even at a higher E:T ratio (8:1) HOZOT exhibited no cytotoxic activity against NHDF whereas it showed more than $90 \%$ cytotoxicity against three tumor cell lines, WiDr, MCF-7 and U2OS. Cytotoxic activity was also robust against these cell lines at a lower E:T ratio (2:1, 37-75\%). These results strongly suggest that HOZOT has a tumor cell-directed cytotoxic capability against a broad range of cancer cells, although further study is necessary for HOZOT's effects on non-neoplastic counterparts.

In a previous study, we demonstrated that HOZOT did not exhibit NK activity, namely no killing against K562 cells (1).
Furthermore, we observed no cytotoxic activity against a variety of hematopoietic tumor cell lines derived from leukemias and lymphomas, such as NALM-6 or BALM-16 (data not shown). No significant cell death was induced during cocultivation with HOZOT when normal hematopoietic cells such as PBMC and macrophages were used as targets (data not shown). These results may also suggest that HOZOT cytotoxically functions for solid tumor cells without significantly affecting non-neoplastic cells.

HOZOT exhibited anti-tumor activity in vivo. In order to examine the practical utility of using HOZOT for in vivo tumor 

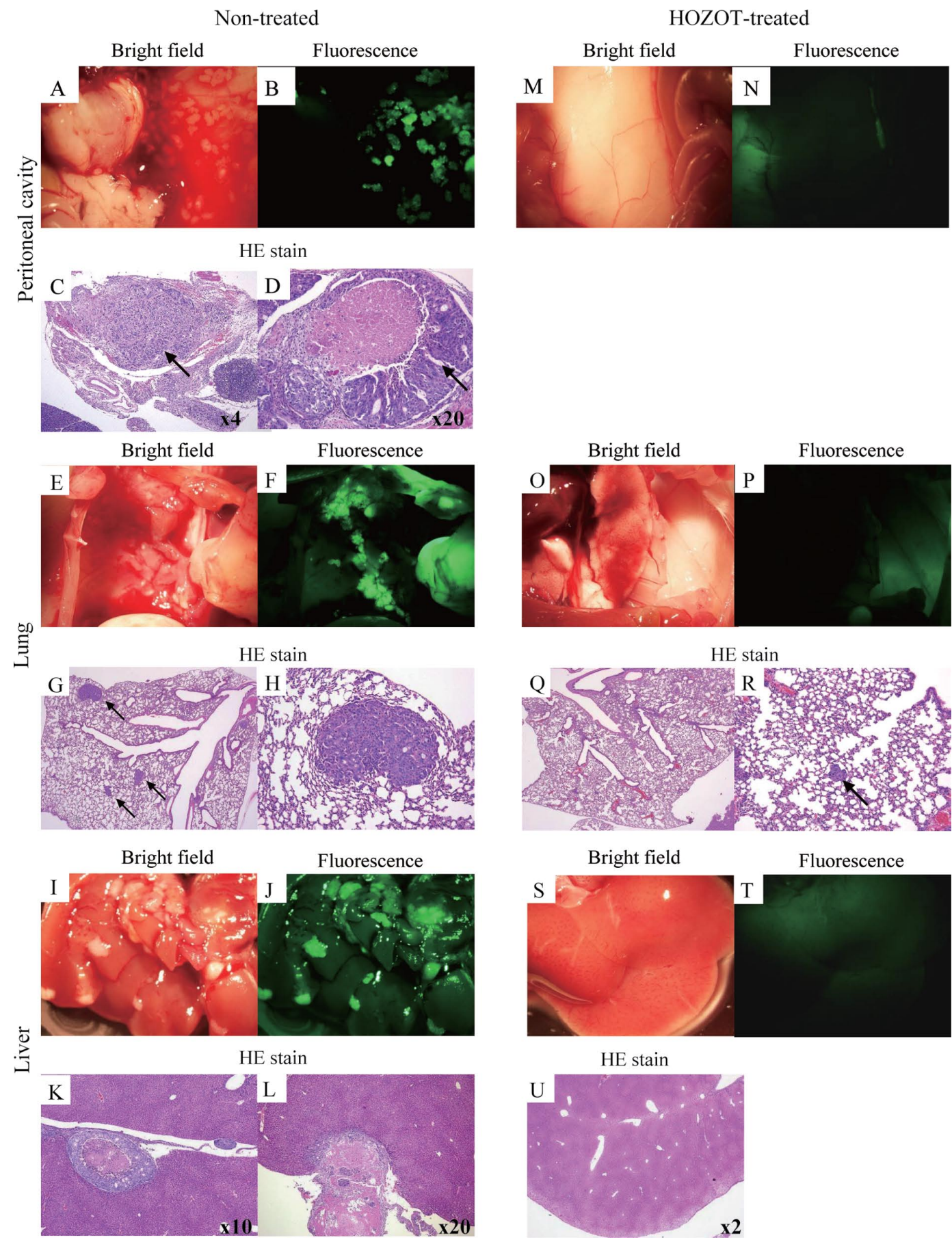

Figure 3. In vivo anti-tumor activity of HOZOT in WiDr-bearing NOD/SCID mouse model. Anti-tumor activity of HOZOT was examined as described in Materials and methods (Experiment 1). Eradication of disseminated WiDr cells was observed in HOZOT-treated mouse (right panels) but not in non-treated mouse (left panels) by examining peritoneal cavity, lung and liver. (A, B, E, F, I and J) Bright field and fluorescence images of peritoneal cavity (A and B) lung (E and F) and liver (I and J) from a non-treated mouse. Tumor cell masses are observed as white spots (A, E and I). These cells were identified as inoculated WiDr by GFP expression (B, F and J). (M, N, O, P, S and T) Bright field and fluorescence images of peritoneal cavity (M and N) lung (O and P) and liver (S and $\mathrm{T}$ ) from non-treated mouse. Tumor cells were not observed. (C, D, G, H, K and L) H\&E-stained paraffin-embedded sections of peritoneal cavity (C and D), lung $(\mathrm{G}$ and $\mathrm{H})$ and liver $(\mathrm{K}$ and $\mathrm{L})$ from non-treated mice. Tumor dissemination was observed in peritoneal cavity $(\mathrm{C}, \mathrm{D}$, black arrow), lung $(\mathrm{G}, \mathrm{H}$, black arrow) and liver (K and L). (Q,R and U) H\&E-stained paraffin-embedded sections of lung (Q and R) and liver (U) from HOZOT-treated mice. Tumor dissemination was not observed in the livers of HOZOT-treated mice (U). Although tumor dissemination was observed in the lungs of HOZOT-treated mice, the size was smaller than in lungs from non-treated mice (black arrow, $\mathrm{G}, \mathrm{H}$ versus $\mathrm{Q}$ and $\mathrm{R}$ ).

killing, we employed a human tumor xenograft mouse model. In this model, NOD/SCID mice were first inoculated intraperitoneally with WiDr-EGFP-9, and treatment with HOZOT was initiated 14 days after WiDr inoculation. In the first protocol, HOZOT was inoculated in the peritoneal cavity of
WiDr-transplanted NOD/SCID mice three times at seven day intervals at an E:T ratio of 2:1. PBS was inoculated in non-treated controls. Fourteen days after the last HOZOT treatment, mice were sacrificed and the intraperitoneal growth of WiDr-EGFP-9 was detected by fluorescence stereomicroscopy. GFP-positive 

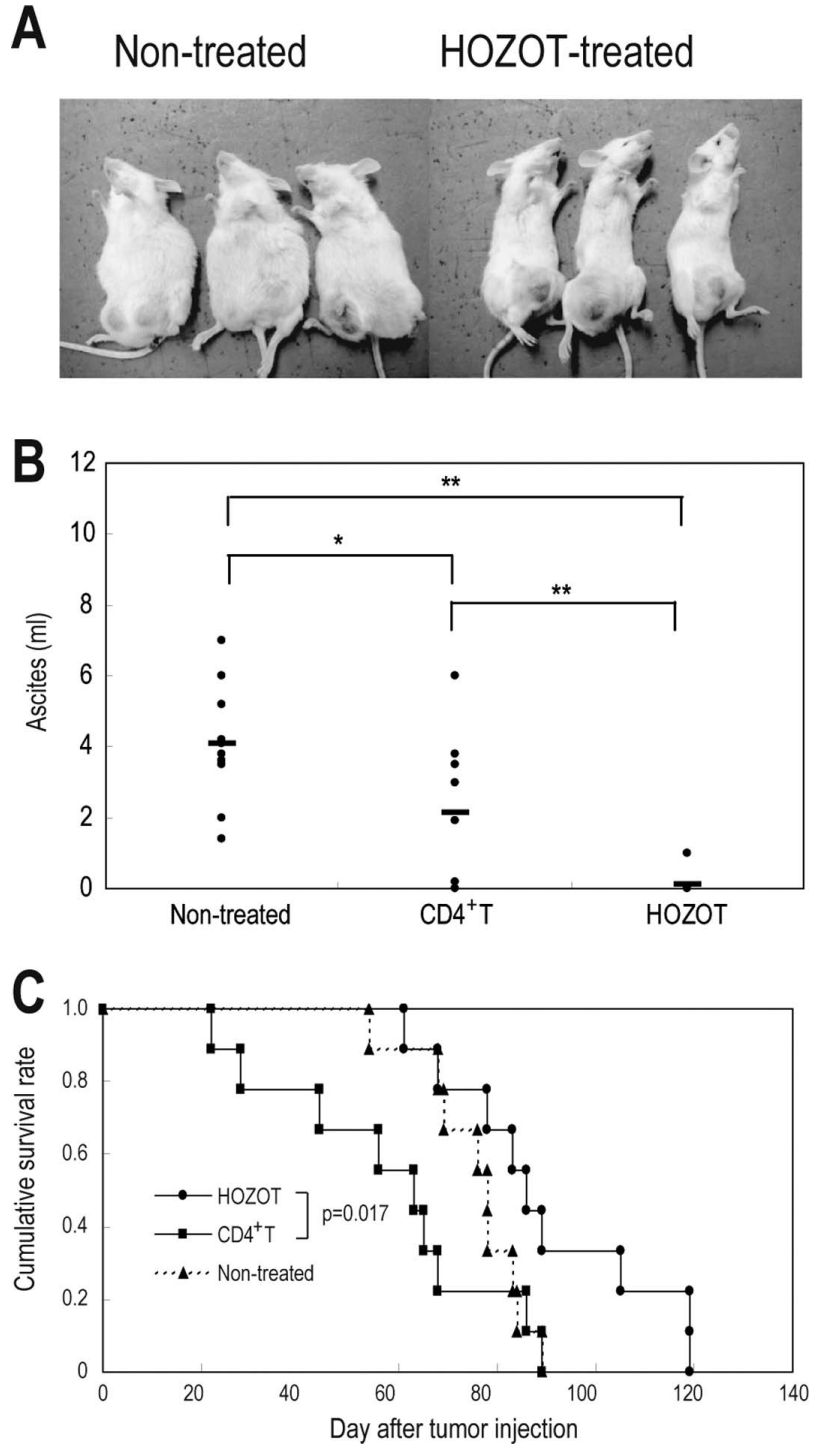

Figure 4. Reduction of ascites accumulation but no effect on lifespan prolongation. Anti-tumor activity of HOZOT was examined as described in Materials and methods (Experiment 2). (A) Macroscopic images of non-treated and HOZOT-treated mice. Marked abdominal distension was observed in all non-treated mice, whereas no abdominal swelling was observed in HOZOTtreated mice. Locally grown tumors in the subcutaneous part of the lower abdomen were observed in all mice with or without HOZOT-treatment. (B) Therapeutic efficacy of HOZOT on the accumulation of ascites induced by intraperitoneally inoculated WiDr. Ascites volume was measured after mice died. Ascites volume was significantly decreased by HOZOT treatment $\left({ }^{* *} \mathrm{p}<0.01\right) . \mathrm{CD}^{+} \mathrm{T}$ cell treatment also decreased ascites volume $\left({ }^{*} \mathrm{p}<0.05\right)$ (C) Overall prolongation of survival by HOZOT treatment in WiDr-bearing mice. HOZOT treatment showed a tendency of prolonged survival, but the difference was not significant when compared with non-treated controls. The effects of HOZOT-treatment in mice were determined by Kaplan-Meier tests.

WiDr cells were almost completely eradicated from the peritoneal cavity of the HOZOT-treated mice (Fig. 3M and N), whereas multiple disseminated lesions of WiDr cells were observed in non-treated mice (Fig. 3A and B). H\&E staining of the mass lesions isolated from mesenterium and peritoneum confirmed that such small nodules consisted entirely of WiDr cell clumps with a histological pattern of moderately differentiated cologenic adenocarcinomas (Fig. 3C and D).
In addition, metastatic foci of WiDr cells were observed not only in the intraperitoneal organs but also in the thoracic cavity and the lungs (Fig. 3E and F). In peritoneal cavity, WiDrEGFP-9 invaded the liver via the surface and the portal area (Fig. 3I and J) in non-treated mice. However, such aggressive metastases were not observed or were markedly reduced in HOZOT-treated mice (Fig. 3O, P, S and T). With respect to the intrapulmonary and intrahepatic metastases, detailed histological examinations of H\&E-stained paraffin-embedded sections of lung and liver were performed for mice with or without HOZOT treatment. As shown in the figures, histology revealed that multiple WiDr metastases to the lung were observed in nontreated mice (Fig. 3G and $\mathrm{H}$ ), although the lesions in the lung were much reduced in number and size in HOZOT-treated mice in comparison with non-treated mice (Fig. 3G and $\mathrm{H}$ vs. $3 \mathrm{Q}$ and R). Similarly, invasive or metastatic foci of WiDr cells in the liver were not observed in HOZOT-treated mice (Fig. 3U), whereas such lesions were prominently observed in non-treated mice (Fig. $3 \mathrm{~K}$ and L). These results demonstrate that HOZOT has a potent tumor-suppressive ability in vivo.

We next evaluated the anti-tumor effect of HOZOT in vivo by employing the tumor-bearing mouse model with peritoneal carcinomatosis. In this protocol, we treated WiDr-transplanted mice with HOZOT four times at seven day intervals at an E:T ratio of 2:1. We used conventional $\mathrm{CD}^{+} \mathrm{T}$ cells instead of HOZOT as a control treatment and PBS as a non-cell treatment control. Mice were monitored until they died of peritoneal carcinomatosis with intraperitoneal tumor growth, and collected tumoral ascites were measured. Remarkably, almost no accumulation of bloody ascites was consistently observed in the HOZOT-treated group (Fig. 4A image and B graph), whereas mice in non-treated and $\mathrm{CD}^{+}{ }^{+} \mathrm{T}$-treated groups showed marked accumulations $(4.08 \mathrm{ml}$ and $2.14 \mathrm{ml}$ on average, $\mathrm{n}=10$, respectively) demonstrating HOZOT treatment was very effective in clearing the peritoneal cavity of tumoral ascites. We next compared the survival rates of HOZOT-treated and non-treated mice. HOZOT treatment tended to prolong survival in the treated mice compared with the non-treated group, but there was no statistically significant difference in survival rates between the two groups (Fig. 4C). This result might be due to the fact that locally grown tumors in the subcutaneous region of the lower abdomen somehow affected their survival (Fig. 4A). However, when compared with the $\mathrm{CD}^{+}{ }^{+}$T-treated group, the HOZOT-treated group did have a significantly higher survival rate $(\mathrm{P}=0.017$, Fig. $4 \mathrm{C})$.

The HOZOT killing mechanism is distinct from conventional $C D 8^{+} C T L, N K$, or NKT cells. Next we examined the molecules involved in cytotoxic mechanisms of HOZOT for human cancer cells. We performed inhibition assays with neutralizing antibodies specific for receptors or cytokines, which are regarded as representative molecules for target recognition or effector function. They included CD8, NKG2D, NKp30, CD1d, TNF- $\alpha$, FasL, TRAIL, and TWEAK. As shown in Fig. $5 \mathrm{~A}$, none of these antibodies significantly blocked the cytotoxic activity of HOZOT against WiDr. In contrast to xenogeneic mouse stromal cell killing (1), anti-CD8 antibody had no effect on WiDr killing, indicating the distinct tumorrecognition mechanisms of HOZOT from $\mathrm{CD}^{+} \mathrm{CTL}$ cells. The results also demonstrated no significant involvement of 


\section{A}

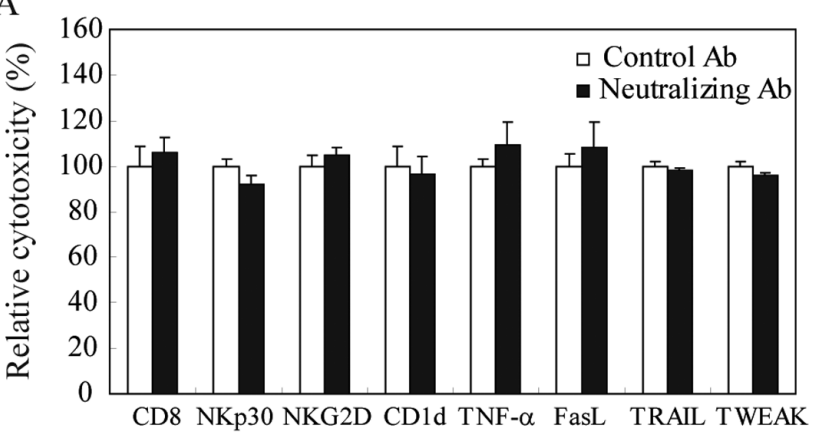

Antibody specificity

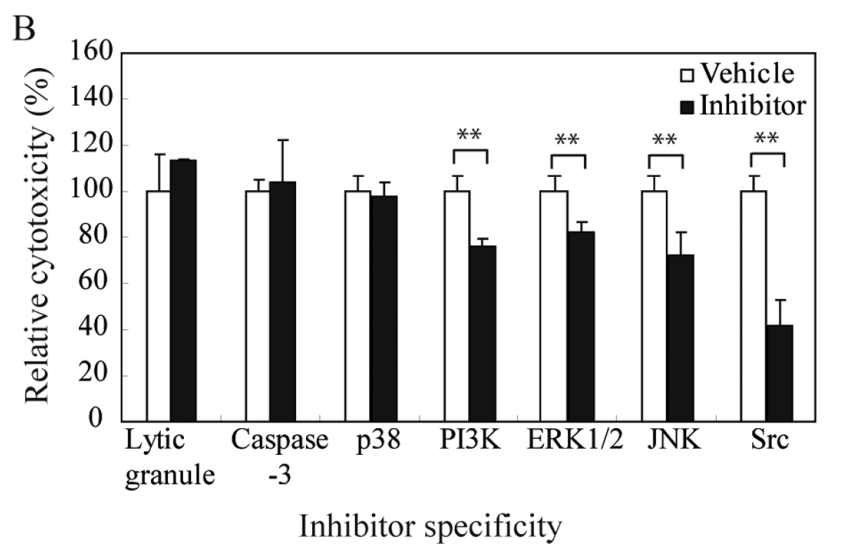

Figure 5. The HOZOT killing mechanisms against tumor cells are distinct from those of CTL, NK or NKT cells. (A) Inhibition assay with antibodies specific for CD8 $(1 \mu \mathrm{g} / \mathrm{ml})$, CD1d $(10 \mu \mathrm{g} / \mathrm{ml})$, NKp30 $(10 \mu \mathrm{g} / \mathrm{ml})$, NKG2D $(10 \mu \mathrm{g} / \mathrm{ml})$, TNF- $\alpha(10 \mu \mathrm{g} / \mathrm{ml})$, FasL $(10 \mu \mathrm{g} / \mathrm{ml})$, TRAIL $(10 \mu \mathrm{g} / \mathrm{ml})$, and TWEAK $(10 \mu \mathrm{g} / \mathrm{ml})$ were performed by adding these antibodies to the cytotoxicity assay of HOZOT against human cancer cells (WiDr). Relative cytotoxic activities are shown in comparison with corresponding negative control antibodies. (B) Inhibition assay with inhibitors specific for p38 $(10 \mu \mathrm{M})$, PI3K $(10 \mu \mathrm{M})$, ERK $(10 \mu \mathrm{M})$, JNK $(10 \mu \mathrm{M})$, or Src family kinase $(10 \mu \mathrm{M})$ and for lytic granule exocytosis $(100 \mathrm{nM})$ or caspase-3 $(25 \mu \mathrm{M})$ were performed by adding them as in (A). Significant blocking effects are shown as ${ }^{* *} \mathrm{p}<0.01$.

NK/NKT recognition molecules, TNF superfamily molecules including TNF- $\alpha$, FasL, TRAIL and TWEAK.

Based on the result of antibody assays, we further performed blocking assays using chemical inhibitors to examine which signaling pathway or effector pathway significantly affected the anti-tumor activity of HOZOT. We used inhibitors for MAP kinase family (p38, ERK1/2, and JNK), PI3K, and Src family kinase as well as for lytic granule exocytosis and caspase-3. As shown in Fig. 5B, the inhibitors for PI3K, ERK1/2, JNK, and Src family kinase showed not perfect but apparently significant inhibitory effects $(17.9 \%, 27.8 \%, 23.4 \%$ and $58.1 \%$ inhibition, respectively), whereas the inhibitors for p38, lytic granules, and caspase- 3 revealed no effects. These results suggest that effector pathways involved in HOZOT's tumor cytotoxicity are obviously distinct from those of other known cytotoxic cells, whose killing mechanisms are dependent on lytic granules and caspase-3.

\section{Discussion}

Previously, we reported a novel type of Treg cell line termed HOZOT $(1,16)$. In our preliminary study, we noticed that
HOZOT exhibited cytotoxic activity to a few tumor cell lines as well as to xenogeneic mouse stromal cells, in addition to typical properties of Tregs such as MLR suppression (17) and IL-10 production (14). To address the question of whether HOZOT would be a powerful anti-tumor cytotoxic tool to develop a novel cellular therapeutics or not, here we first screened a panel of twenty human tumor cell lines with diverse origins. Consequently, it was found that HOZOT functions as a potent cytotoxic effector for a wide spectrum of tumor cells.

In order to investigate the in vivo efficacy of HOZOT's anti-tumor activity, we utilized a human/mouse chimeric model where human effector and human origin target cells were xenografted into NOD/SCID mice. Highly efficient anti-tumor effects were observed in HOZOT-treated mice which demonstrated almost complete eradication of inoculated WiDr cells in the peritoneal cavity and no accumulation of tumor ascites. In clinical oncology of cancers at advanced stages, especially the advanced stage caused by aggressive carcinomas mainly from digestive organs, it is important to manage cancer cell dissemination in the peritoneal/thoracic cavity and to reduce the accumulation of tumor ascites $(18,19)$. Taking these issues into consideration, our results emphasize the promising clinical utility of HOZOT against advanced stage colon cancer. HOZOT treatment did not clearly provide statistically significant prolonged survival in a NOD/SCID mouse model. However, this is probably due to WiDr cells leaking into subcutaneous tissues at the time of intraperitoneal inoculation (Fig. 4A), which gradually enlarged at the subcutaneous site and resulted in eventual death of the mice. Despite this problem, our in vivo model was encouraging and further developing HOZOT as an anti-cancer therapy remains promising.

We investigated the cytotoxic mechanism of HOZOT against human tumor cells. There are some reports mentioning Tregs exhibiting cytotoxic activity. Grossman et al reported that when stimulated with anti-CD3/CD46 antibodies, both naturally occurring and adaptive Tregs exhibited cytotoxic activity against autologous normal target cells through CD8/ TCR-independent and perforin-dependent, NK-like mechanisms (9). Also, $\mathrm{CD} 8^{+}$Tregs induced by allogeneic CD40-activated plasmacytoid dendritic cells exhibited cytotoxic activity against allogenic target cells (10). In both cases, the Treg's cytotoxicity against normal cells was associated with their suppression mechanisms. Therefore, the direct tumor-specific cytotoxicity of HOZOT provides a sharp contrast to the autologous or allogenic cytotoxicity of $\mathrm{CD}^{+}$or $\mathrm{CD} 8^{+}$cytotoxic Tregs. Moreover, even HOZOT itself might use distinct killing mechanisms for different target cells, for example, mouse versus human cells. When killing mouse cells, HOZOT behaves like CTL and cytotoxicity can be blocked with anti-CD8 antibodies. On the other hand, none of the antibodies specific for CD8, NK receptors, or CDld could inhibit the cytotoxic activity of HOZOT against human cancer cells. Noticeably, treatment with inhibitors for lytic granule exocytosis and caspase-3, which evidently blocked HOZOT's killing against mouse stromal cells, also resulted in no inhibition of its killing against WiDr. In addition, the experiment employing chemical inhibitors, which showed the involvement of Src family kinase and other MAP kinases, may provide certain clues to this type of tumorspecific killing. Taking these results all together, they repeatedly indicate that HOZOT's cytotoxicity is mediated through different 
mechanisms from those of other known killer cells at the target recognition phase as well as the effector phase. To explain this novel killing mechanism from another aspect except antibodies and molecular reagents, we would also like to highlight the unique behavior of HOZOT, 'cell-in-cell activity', which has been recently reported as a phenomenon of host tumor cell death induced by intracellularly penetrated HOZOT (20). After this internalization of HOZOT, apoptotic cell death of tumor cells was induced. This activity must be one of effector mechanisms of anti-tumor activity of HOZOT against WiDr because HOZOT could internalize to WiDr. But we consider that other effector mechanisms must exist because frequency of HOZOT internalization into WiDr was only about $10 \%$.

In conclusion, we demonstrated that a cytotoxic Treg cell line, HOZOT, exerted potent anti-tumor cytotoxicity against a broad range of human tumor types. Although the concrete details still remain unclear, its cytopathic mechanisms seem to be distinct from other cytotoxic cells such as CTL, NK or NKT cells. We also demonstrated the in vivo efficacy of HOZOT's anti-tumor activity in a tumor-bearing NOD/SCID mouse model. Although further investigation of anti-tumor mechanism is necessary, HOZOT has potential to be a novel powerful immunotherapeutic effector against aggressive malignancies and may be an important tool for exploring unknown cytotoxic mechanisms of human $\mathrm{T}$ cells.

\section{Acknowledgements}

We thank Dr Toshitada Takahashi (Comprehensive Health Science Center, Aichi Health Promotion Foundations, Nagoya) and Dr Kunzo Orita (Hayashibara Biochemical Laboratories Inc.) for critical reviews and helpful discus-sions. This study was partially supported by a grant from the Ministry of Education, Culture, Sports, Science and Technology FY2006 'Creation of Innovation Centers for Advanced Interdisciplinary Research Areas' Scheme in Japan.

\section{References}

1. Nakamura S, Suzuki M, Sugimoto A, et al: IL-2-independent generation of FOXP3(+)CD4(+)CD8(+)CD25(+) cytotoxic regulatory $\mathrm{T}$ cell lines from human umbilical cord blood. Exp Hematol 35: 287-296, 2007.

2. Leen AM, Rooney CM and Foster AE: Improving T cell therapy for cancer. Annu Rev Immunol 25: 243-265, 2007.

3. Kabelitz D, Wesch D and He W: Perspectives of gammadelta T cells in tumor immunology. Cancer Res 67: 5-8, 2007.
4. Terme M, Ullrich E, Delahaye NF, Chaput $\mathrm{N}$ and Zitvogel L: Natural killer cell-directed therapies: moving from unexpected results to successful strategies. Nat Immunol 9: 486-494, 2008.

5. Taniguchi M, Harada M, Kojo S, Nakayama T and Wakao H: The regulatory role of Valpha14 NKT cells in innate and acquired immune response. Annu Rev Immunol 21: 483-513, 2003.

6. Ha TY: The role of regulatory T cells in cancer. Immune Netw 9: 209-235, 2009

7. Vivier E, Tomasello E, Baratin M, Walzer T and Ugolini S: Functions of natural killer cells. Nat Immunol 9: 503-510, 2008.

8. Terabe M and Berzofsky JA: The role of NKT cells in tumor immunity. Adv Cancer Res 101: 277-348, 2008.

9. Grossman WJ, Verbsky JW, Barchet W, Colonna M, Atkinson JP and Ley TJ: Human T regulatory cells can use the perforin pathway to cause autologous target cell death. Immunity 21 : 589-601, 2004

10. Zheng J, Liu Y, Qin G, et al: Efficient induction and expansion of human alloantigen-specific CD8 regulatory $\mathrm{T}$ cells from naive precursors by CD40-activated B cells. J Immunol 183: 3742-3750, 2009.

11. Tanaka J, Toubai T, Tsutsumi Y, et al: Cytolytic activity and regulatory functions of inhibitory NK cell receptor-expressing $T$ cells expanded from granulocyte colony-stimulating factormobilized peripheral blood mononuclear cells. Blood 104: 768-774, 2004

12. Young KJ, DuTemple B, Phillips MJ and Zhang L: Inhibition of graft-versus-host disease by double-negative regulatory T cells. J Immunol 171: 134-141, 2003.

13. Otani T, Nakamura S, Inoue T, et al: Erythroblasts derived in vitro from embryonic stem cells in the presence of erythropoietin do not express the TER-119 antigen. Exp Hematol 32: 607-613, 2004.

14. Tsuji-Takayama K, Suzuki M, Yamamoto M, et al: The production of IL-10 by human regulatory T cells is enhanced by IL-2 through a STAT5-responsive intronic enhancer in the IL-10 locus. J Immunol 181: 3897-3905, 2008.

15. Fukazawa T, Matsuoka J, Naomoto Y, Maeda Y, Durbin ML and Tanaka N: Malignant pleural mesothelioma-targeted CREBBP/ EP300 inhibitory protein 1 promoter system for gene therapy and virotherapy. Cancer Res 68: 7120-7129, 2008.

16. Harashima A, Toraya $\mathrm{T}$, Okochi $\mathrm{A}$, et al: Interleukin-8 and RANTES are signature cytokines made by HOZOT, a new type of regulatory T cells. Mol Immunol 46: 3310-3319, 2009.

17. Suzuki M, Sugimoto A, Harashima A, et al: Novel mechanisms of suppressor activity exhibited by cytotoxic regulatory $\mathrm{T}$ cell lines, HOZOT. Exp Hematol 37: 92-100, 2009.

18. Verheul HM, Hoekman K, Jorna AS, Smit EF and Pinedo HM: Targeting vascular endothelial growth factor blockade: ascites and pleural effusion formation. Oncologist 5 (Suppl 1): 45-50, 2000.

19. Smith EM and Jayson GC: The current and future management of malignant ascites. Clin Oncol (R Coll Radiol) 15: 59-72, 2003.

20. Takeuchi M, Inoue T, Otani T, Yamasaki F, Nakamura S and Kibata M: Cell-in-cell structures formed between human cancer cell lines and the cytotoxic regulatory T-cell line HOZOT. J Mol Cell Biol 2: 139-151, 2010. 